\title{
PRIMITIVE ELEMENTS OF GALOIS EXTENSIONS OF FINITE FIELDS
}

\author{
ISAO KIKUMASA AND TAKASI NAGAHARA
}

(Communicated by Louis J. Ratliff, Jr.)

Dedicated to Professor Nobuo Nobusawa on his sixtieth birthday

\begin{abstract}
As is well known, $\mathrm{N}_{q}(n)=(1 / n) \sum_{d \mid n} \mu(d) q^{n / d}$ coincides with the number of monic irreducible polynomials of $\operatorname{GF}(q)[X]$ of degree $n$. In this note we discuss the curve $n \mathrm{~N}_{X}(n)$ and the solutions of equations $n \mathrm{~N}_{X}(n)=b$ $(b \geq n)$. As a corollary of these results, we present a necessary and sufficient arithmetical condition for $R / K$ to have a primitive element.
\end{abstract}

\section{INTRODUCTION}

Throughout this paper, $K$ means a finite field, and all ring extensions of $K$ are assumed to be commutative and have an identity that is contained in $K$. Moreover, all Galois extensions mean that in the sense of [1]. A Galois extension $R / K$ is called simple if $R$ is $K$-algebra isomorphic to a factor ring $K[X] /(h)$ for some polynomial $h$ in $K[X]$, that is, $R / K$ has a primitive element.

In $[4,6,7]$ and etc., the authors made some studies on primitive elements of Galois extensions from several angles. On the other hand, the simplicity of separable extensions was recently discussed by J. -D. Thérond [14] in some directions. But, conditions studied in [14] are necessary and sufficient conditions so that "all" separable extensions of a semilocal ring have primitive elements. Hence, these conditions are not always applicable to discuss whether a given Galois extension is simple or not.

The purpose of this note is to study the solutions of a certain equation, which is concerned with finite fields and, using these results, to present arithmetical conditions for the simplicity of Galois extensions over $K$.

In $\S 1$ we consider a polynomial of degree $m$ :

$$
\mathrm{N}_{X}(m)=(1 / m) \sum_{d \mid m} \mu(d) X^{m / d},
$$

where $\mu$ is the Moebius function on the set of natural numbers. As in [9], for a finite field $\operatorname{GF}(q)$ with $q$ a power of a prime number, $\mathrm{N}_{q}(m)$ is the number of monic irreducible polynomials of $\operatorname{GF}(q)[X]$ of degree $m$. The aim

Received by the editors May 30, 1990 and, in revised form, December 12, 1990.

1980 Mathematics Subject Classification (1985 Revision). Primary 13B05; Secondary 13B25, $12 \mathrm{E} 12$. 
of this section is to pursue the curve of $m \cdot \mathrm{N}_{X}(m)$ and to study the solutions of equations $m \cdot \mathrm{N}_{X}(m)=b \quad(b \geq m)$ on the interval $[1, \infty)$.

In $\S 2$ we present a necessary and sufficient condition for the simplicity of Galois extensions of $K$. In this discussion, the solutions of the equations in the above play an important role.

In what follows, given a $K$-algebra $R$ and a set $S$, we use the following conventions: $[R: K]$ denotes the rank of $K$-module $R, l(R)$ the length of composition series of $R$-module $R$, and $|S|$ the cardinal number of $S$. Further, by $\mathbf{N}$ and $\mathbf{R}$, we denote the set of positive integers and the set of real numbers respectively.

\section{AN ALgebraic EQUATION CONCERNed With $\mathrm{N}_{q}(a)$}

Let

$$
a=p_{1}^{\alpha_{1}} p_{2}^{\alpha_{2}} \cdots p_{n}^{\alpha_{n}},
$$

where $n_{1}, \alpha_{1}, \alpha_{2}, \ldots, \alpha_{n} \in \mathbf{N}$ and $p_{1}, p_{2}, \ldots, p_{n}$ are distinct prime numbers. Then, we set

$$
\begin{aligned}
& f(X)=\sum_{\substack{1 \leq e_{1}<e_{2}<\cdots<e_{i} \leq n, 0 \leq i \leq n \\
g(X)}}(-1)^{n-i} X^{p_{1} p_{2} \cdots p_{n}}-f(X),
\end{aligned}
$$

where $p_{e_{1}} p_{e_{2}} \cdots p_{e_{i}}=1$ if $i=0$. One will easily see that the number of terms in $f(X)$ is $\sum_{i=0}^{n}\left(\begin{array}{c}n \\ i\end{array}\right)=2^{n}$.

Now, we consider the equation

$$
f(x)=a \mathrm{~N}_{q}(a) .
$$

Then, as is shown in $\S 2, \xi:=q^{a /\left(p_{1} p_{2} \cdots p_{n}\right)}$ is a solution of this equation, that is, $f(\xi)=a \mathrm{~N}_{q}(a)$. Moreover, for $K=\mathrm{GF}(q)$, a $G$-Galois extension $R / K$ with $a=|G| / l(R)$ is simple if and only if $|G| \leq a \mathrm{~N}_{q}(a)=f(\xi)$.

In this section, we study the solutions of the algebraic equation

$$
f(x)=b \quad(a \leq b \in \mathbf{N}) .
$$

First we prove the following theorem, which plays an important role in our study.

Theorem 1.1. Let $f(X)$ and $g(X)$ be as in (*). Then

(1) $f(1)=0$ and $g(1)=1$.

(2) $f(x)$ and $g(x)$ are strictly increasing on the interval $[1, \infty)$.

Proof. It is obvious that

$$
f(1)=\sum_{i=0}^{n}(-1)^{n-i}\left(\begin{array}{l}
n \\
i
\end{array}\right)=(1-1)^{n}=0
$$

and so $g(1)=1-f(1)=1$. Hence we prove (2). 
For the base $e$ of the natural logarithm, we set

(a)

$$
\begin{aligned}
& h_{0}(t)=e^{t} \quad(t>0) \\
& h_{1}(t)=h_{0}\left(p_{1} t\right)-h_{0}(t) \\
& \ldots \ldots \ldots \ldots \ldots \ldots \ldots \ldots \ldots \ldots \ldots \ldots \ldots \ldots \ldots \\
& h_{i+1}(t)=h_{i}\left(p_{i+1} t\right)-h_{i}(t) \\
& \ldots \ldots \ldots \ldots \ldots \ldots \ldots \ldots \ldots \\
& h_{n}(t)=h_{n-1}\left(p_{n} t\right)-h_{n-1}(t) .
\end{aligned}
$$

Then, it is easily seen that

$$
\begin{gathered}
h_{n}(t)=h_{0}\left(p_{1} \cdots p_{n} t\right)-\sum_{i=0}^{n-2} h_{i}\left(p_{i+2} \cdots p_{n} t\right)-h_{n-1}(t), \\
h_{n}(t)=\sum_{1 \leq e_{1}<e_{2}<\cdots<e_{i} \leq n, 0 \leq i \leq n}(-1)^{n-i}\left(e^{t}\right)^{p_{e_{1}} \cdots p_{e_{i}}},
\end{gathered}
$$

where $p_{e_{1}} \cdots p_{e_{i}}=1$ when $i=0$.

Let $\Omega_{0}$ be the set of all strictly increasing functions $h(z)$ with $h(z)>0$ on the interval $] 0, \infty)$ and, similarly, $\Omega_{1}$ on the interval $\left.] 1, \infty\right)$. Clearly, $h_{0}^{(m)}(t)=e^{t} \in \Omega_{0}$ for $m=0,1,2, \ldots$. Assume that $0 \leq i \leq n-1$ and $h_{i}^{(m)}(t) \in \Omega_{0}$ for $m=0,1,2, \ldots$. Then for any $t>0$ and each $m \geq 0$, we have $h_{i}^{(m)}\left(p_{i+1} t\right)>h_{i}^{(m)}(t)$ and so

$$
h_{i+1}^{(m)}(t)=p_{i+1}^{m} h_{i}^{(m)}\left(p_{i+1} t\right)-h_{i}^{(m)}(t)>0 .
$$

This means that $h_{i+1}^{(m)}(t) \in \Omega_{0}$ for $m=0,1,2, \ldots$. Hence, we get

$$
h_{i}^{(m)}(t) \in \Omega_{0} \quad \text { for } 0 \leq i \leq n \text { and } m \geq 0 .
$$

In particular, $h_{n}(t) \in \Omega_{0}$. We note here that the function $t=\log _{e} x$ belongs to $\Omega_{1}$. Since $f(X)=h_{n}\left(\log _{e} X\right)$ by (c), we obtain $f(x) \in \Omega_{1}$. Moreover, by (d),

$$
\sum_{i=0}^{n-2} h_{i}\left(p_{i+2} \cdots p_{n} t\right)+h_{n-1}(t) \in \Omega_{0} .
$$

This implies that $g(x) \in \Omega_{1}$ by (b). Combining these with the fact that $f(x)$ and $g(x)$ are continuous on $(-\infty,+\infty)$, we have assertion (2).

Corollary 1.2. Let $g(X)$ be as in $(*)$. Then

$$
x \leq g(x) \leq \sum_{i=1}^{n} x^{\left(p_{1} p_{2} \cdots p_{n}\right) / p_{i}} \quad \text { for } x \geq 1 .
$$

In particular, if $n=1$ then $x=g(x)$.

Proof. Let $n=1$. Then obviously $x=g(x)$ and so we assume that $n \geq 2$.

Let $h_{i} \quad(0 \leq i \leq n)$ be as in the proof of Theorem 1.1. Then, as is easily seen, we have

$$
0 \leq h_{i}(t) \leq h_{0}\left(p_{1} p_{2} \cdots p_{i} t\right) \quad \text { for } t \geq 0 .
$$

Hence, it follows that

$$
\begin{aligned}
0 \leq h_{i}\left(p_{i+2} \cdots p_{n} t\right) & \leq h_{0}\left(p_{1} \cdots p_{i} p_{i+2} \cdots p_{n} t\right) \\
& =\left(e^{t}\right)^{\left(p_{1} p_{2} \cdots p_{n}\right) / p_{i+1}} \quad(0 \leq i \leq n-2),
\end{aligned}
$$


and

$$
0 \leq h_{n-1}(t) \leq\left(e^{t}\right)^{\left(p_{1} p_{2} \cdots p_{n}\right) / p_{n}} .
$$

Thus, by (b), we obtain that

$$
\begin{aligned}
g\left(e^{t}\right) & =\sum_{i=0}^{n-2} h_{i}\left(p_{i+2} \cdots p_{n} t\right)+h_{n-1}(t) \\
& \leq \sum_{i=0}^{n-2}\left(e^{t}\right)^{\left(p_{1} \cdots p_{n}\right) / p_{i+1}}+\left(e^{t}\right)^{\left(p_{1} \cdots p_{n}\right) / p_{n}}=\sum_{i=1}^{n}\left(e^{t}\right)^{\left(p_{1} \cdots p_{n}\right) / p_{i}}
\end{aligned}
$$

Next, by (b) again, we have

$$
\begin{aligned}
g\left(e^{t}\right) & =h_{0}\left(p_{2} \cdots p_{n} t\right)+\left(\sum_{i=0}^{n-2} h_{i}\left(p_{i+2} \cdots p_{n} t\right)+h_{n-1}(t)\right) \\
& =h_{0}\left(p_{2} \cdots p_{n} t\right)+C=\left(e^{t}\right)^{p_{2} \cdots p_{n}}+C,
\end{aligned}
$$

where $C \geq 0$. Then $g\left(e^{t}\right)-e^{t}=\left(\left(e^{t}\right)^{p_{2} \cdots p_{n}}-e^{t}\right)+C \geq 0$ for $t \geq 0$. Setting $x=e^{t} \quad(x \geq 1)$, we obtain our assertion.

Corollary 1.3. Let $f(X)$ and $g(X)$ be given as $(*)$.

(1) If $x \geq 2$ then $f(x) \geq g(x)$.

(2) If $0 \leq x \leq 1$ then $|\bar{f}(x)|<2^{n}$.

(3) For $b \in \mathbf{R}$ with $b \geq 2^{n}$, the equation $f(x)=b$ has a solution in ] $1, \infty)$, which is unique in $10, \infty)$.

Proof. (1) If $n=1$ then $f(x)-g(x)=x^{p_{1}}-2 x=x\left(x^{p_{1}-1}-2\right) \geq 0$ for $x \geq 2$.

Let $n \geq 2$ and $\alpha=p_{1} p_{2} \cdots p_{n}$. Without loss of generality, we can assume that $p_{1}<p_{2}<\cdots<p_{n}$. Then $\alpha \geq 2 p_{2} p_{3} \cdots p_{n} \geq p_{2} p_{3} \cdots p_{n}+2$. Hence the degree of the leading term of $g(x)$ is not greater than $\alpha-2$. Since all the terms in $g(x)$ have distinct degrees, we have

$$
g(x) \leq x^{\alpha-2}+x^{\alpha-3}+\cdots+x+1
$$

and so,

$$
\begin{aligned}
f(x)-g(x) & =x^{\alpha}-2 g(x) \\
& \geq x^{\alpha}-2\left(x^{\alpha-2}+x^{\alpha-3}+\cdots+x+1\right) \\
& \geq x^{\alpha}-x\left(x^{\alpha-2}+x^{\alpha-3}+\cdots+x+1\right) \\
& >x^{\alpha}-\left(x^{\alpha}-1\right) /(x-1) \\
& =\left(x^{\alpha}(x-2)+1\right) /(x-1)>0 \quad(x \geq 2) .
\end{aligned}
$$

Thus we obtain $f(x) \geq g(x)$ for $x \geq 2$.

(2) Obviously $f(0)=0$ and, by Theorem $1.1(1), f(1)=0$. Hence we can assume that $0<x<1$. Then the absolute value of each term in $f(x)$ is less than 1 and the number of terms in $f(x)$ is $2^{n}$. Thus we have $|f(x)|<2^{n}$.

(3) This is a direct consequence of (2), Theorem 1.1(2) and $\lim _{x \rightarrow \infty} f(x)=$ $\infty$.

The following theorem is one of our main results in this note. 
Theorem 1.4. Let $f(X)$ and $g(X)$ be defined by $(*)$, and let $b \in \mathbf{N}$ with $b \geq p_{1} p_{2} \cdots p_{n}$. Then, the equation

$$
f(x)=b \quad(x>0)
$$

has a unique solution. Furthermore, for the solution $x_{0}$ of the equation $(* *)$, the following inequality holds:

$$
1<x_{0} \leq g\left(x_{0}\right) \leq b .
$$

Proof. Since $b \geq p_{1} p_{2} \cdots p_{n} \geq 2^{n}$, the equation $f(x)=b \quad(x>0)$ has a unique solution $x_{0}$ with $x_{0}>1$ by Corollary 1.3. Further, it follows immediately from Corollary 1.2 that $x_{0} \leq g\left(x_{0}\right)$. Let $\alpha$ be a real number with $1 / b<$ $\alpha \leq 1$. Then, since the equation $g(x)=\alpha b(x>1)$ has a unique solution by Theorem 1.1, we write this by $x_{1}$. Moreover, let $x_{2}$ be the root of the equation $x^{p_{1} p_{2} \cdots p_{n}}=(\alpha+1) b=g\left(x_{1}\right)+b$. Suppose that $g\left(x_{2}\right) \leq \alpha b$. Then, by Theorem 1.1(2), we have $x_{2} \leq x_{1}$ and so

$$
f\left(x_{0}\right)=b=x_{2}^{p_{1} p_{2} \cdots p_{n}}-g\left(x_{1}\right) \leq x_{1}^{p_{1} p_{2} \cdots p_{n}}-g\left(x_{1}\right)=f\left(x_{1}\right) .
$$

In virtue of Theorem 1.1 again, we get $x_{0} \leq x_{1}$ and whence $g\left(x_{0}\right) \leq g\left(x_{1}\right)=$ $\alpha b \leq b$. Hence, to prove the theorem, all we must do is to show that the inequality $g\left(x_{2}\right) \leq \alpha b$ holds for some $\alpha$ in $\left.] 1 / b, 1\right]$. In case $b \geq 2 n^{2}$, take 1 as $\alpha$. Then it follows from Corollary 1.2 that

$$
\begin{aligned}
g\left(x_{2}\right) & \leq \sum_{i=1}^{n} x_{2}^{\left(p_{1} p_{2} \cdots p_{n}\right) / p_{i}}=\sum_{i=1}^{n}((\alpha+1) b)^{1 / p_{i}} \\
& \leq n((\alpha+1) b)^{1 / 2}=\sqrt{2 n^{2} b} \leq \sqrt{b \cdot b}=b=\alpha b, \quad \text { for } \alpha=1 .
\end{aligned}
$$

If $b<2 n^{2}$ then $\left(n, p_{1} p_{2}, b\right)=(2,6,6)$ or $(2,6,7)$ because $b \geq p_{1} p_{2} \cdots p_{n} \geq$ $2 \cdot 3 \cdots n(n+1)$. Let $n=2$ and $p_{1} p_{2}=b=6$. Then, putting $\alpha=2 / 3, x_{2}^{6}=10$ and so

$$
\begin{aligned}
g\left(x_{2}\right) & =x_{2}^{3}+x_{2}^{2}-x_{2}=\sqrt{10}+\sqrt[3]{10}-\sqrt[6]{10} \\
& <3.2+2.2-1.4=4=\alpha b .
\end{aligned}
$$

Similarly, in case that $n=2, p_{1} p_{2}=6$, and $b=7$, we put $\alpha=6 / 7$. Then

$$
g\left(x_{2}\right)=\sqrt{13}+\sqrt[3]{13}-\sqrt[6]{13}<4+3-1=\alpha b .
$$

This completes the proof.

Remark 1.5. In the notation of Theorem 1.4, we denote the solution $x_{0}$ of the equation $(* *)$ by $x_{0}(a, b)$. Moreover, we set

$$
\varepsilon(a, b)=g\left(x_{0}(a, b)\right) / b .
$$

Then

(1) $0<\varepsilon(a, b) \leq 1$.

(2) If $b=p_{1}^{\beta_{1}} p_{2}^{\beta_{2}} \cdots p_{n}^{\beta_{n}}$ where $\beta_{i} \in \mathbf{N}(i=1,2, \ldots, n)$ then

$$
x_{0}(a, b)=x_{0}(b, b) \quad \text { and } \quad \varepsilon(a, b)=\varepsilon(b, b) .
$$

In addition, we put $\varepsilon(1, b)=0$. 
Example 1.6. Let $a=2 \cdot 3$ and $b=2^{3} \cdot 3^{2}$. Then the equation $(* *)$ in Theorem 1.4 is

$$
x^{6}-x^{3}-x^{2}+x-72=0 \quad(x>0) .
$$

As is easily seen, the solution $x_{0}(6,72)$ of this equation satisfies

$$
2.0902<x_{0}(6,72)<2.0903 \text {. }
$$

Moreover, from this inequality, we obtain

$$
0.158<\varepsilon(6,72)<0.159 \text {. }
$$

\section{Primitive elements of Galois extensions of finite fields}

Throughout this section, let $q$ be a power of a prime number. We begin this section with the following lemma, which is fundamental.

Lemma 2.1 [7, Theorem 1.6]. Let $R$ be a Galois extension of rank $b$ of $\operatorname{GF}(q)$. Then the extension $R / \mathrm{GF}(q)$ is simple if and only if $l(R) \leq \mathrm{N}_{q}(b / l(R))$.

Combining this lemma with the results in $\S 1$, we have the following theorem, which is a generalization of [5, Proposition 1].

Theorem 2.2. Let $R$ be a G-Galois extension of $\operatorname{GF}\left(p^{s}\right), b=|G|$, and $a=$ $b / l(R)$. Then the extension $R / \mathrm{GF}\left(p^{s}\right)$ is simple if and only if

$$
l(R) \leq b s /\left(\log _{p} b+\log _{p}(1+\varepsilon(a, b))\right),
$$

where $\varepsilon(a, b)$ is the constant given in $\S 1$, which depends only on $b$ and the prime divisors of $a$. In particular, when any prime divisor of $b$ divides $a$, the extension $R / \mathrm{GF}\left(p^{s}\right)$ is simple if and only if

$$
l(R) \leq b s /\left(\log _{p} b+\log _{p}(1+\varepsilon(b, b))\right) .
$$

Proof. Put $q=p^{s}$. In case $l(R)=b$, it follows from Lemma 2.1 and the fact $\mathrm{N}_{q}(1)=q$ that $R / K$ is simple if and only if $l(R) \leq q$, which is equivalent to that $l(R) \leq b s /\left(\log _{p} b+\log _{p}(1+\varepsilon(a, b))\right)$. Hence we assume that $l(R) \neq b$. Let $a=p_{1}^{\alpha_{1}} p_{2}^{\alpha_{2}} \cdots p_{n}^{\alpha_{n}}$ where $n, \alpha_{1}, \alpha_{2}, \ldots, \alpha_{n} \in \mathbf{N}$, and $p_{1}, p_{2}, \ldots, p_{n}$ are distinct prime numbers. Moreover, let $f(X)$ be given as $(*)$. Then,

$$
\begin{aligned}
a \mathrm{~N}_{q}(a)= & \sum_{d \mid a} \mu(d) q^{a / d} \\
= & q^{a}-q^{a / p_{1}}-q^{a / p_{2}}-\cdots+q^{a\left(p_{1} p_{2}\right)}+q^{a /\left(p_{1} p_{3}\right)}+\cdots \\
& +\cdots+(-1)^{i} q^{a\left(p_{e_{1}} p_{e_{2}} \cdots p_{p_{i}}\right)}+\cdots+(-1)^{n} q^{a /\left(p_{1} p_{2} \cdots p_{n}\right)} \\
= & f\left(q^{a /\left(p_{1} p_{2} \cdots p_{n}\right)}\right),
\end{aligned}
$$

where $1 \leq e_{1}<e_{2}<\cdots<e_{i} \leq n$. We have already noted that $f(x)$ is strictly increasing on $x>1$ by Theorem 1.1 and $x_{0}(a, b)>1$ by Theorem 1.4. Hence the inequality $f\left(x_{0}(a, b)\right) \leq f\left(q^{a /\left(p_{1} p_{2} \cdots p_{n}\right)}\right)$ is equivalent to $x_{0}(a, b) \leq$ $q^{a /\left(p_{1} p_{2} \cdots p_{n}\right)}$. Since $l(R) \leq \mathrm{N}_{q}(a)$ if and only if $b \leq a \mathrm{~N}_{q}(a)$, it follows from 
Lemma 2.1 that

$$
\begin{aligned}
R / K \text { is simple } & \Longleftrightarrow b \leq f\left(q^{a /\left(p_{1} p_{2} \cdots p_{n}\right)}\right) \\
& \Longleftrightarrow f\left(x_{0}(a, b)\right) \leq f\left(q^{a /\left(p_{1} p_{2} \cdots p_{n}\right)}\right) \\
& \Longleftrightarrow x_{0}(a, b) \leq q^{a /\left(p_{1} p_{2} \cdots p_{n}\right)} \\
& \Longleftrightarrow \log _{q}\left(x_{0}(a, b)^{p_{1} p_{2} \cdots p_{n}}\right) \leq a \\
& \Longleftrightarrow \log _{q}\left(b+g\left(x_{0}(a, b)\right)\right) \leq a \\
& \Longleftrightarrow b / \log _{q}\left(b+g\left(x_{0}(a, b)\right)\right) \geq b / a=l(R) .
\end{aligned}
$$

Since $q=p^{s}$, we have

$$
\begin{aligned}
b / \log _{q}\left(b+g\left(x_{0}(a, b)\right)\right) & =b /\left(\log _{p}\left(b+g\left(x_{0}(a, b)\right)\right) / \log _{p} q\right) \\
& =b s /\left(\log _{p} b+\log _{p}\left(1+g\left(x_{0}(a, b)\right) / b\right)\right) \\
& =b s /\left(\log _{p} b+\log _{p}(1+\varepsilon(a, b))\right) .
\end{aligned}
$$

Combining this with the previous equivalence relation, we obtain the first part of our assertion. The second assertion follows from Remark 1.5(2).

The following is a corollary of the above theorem, and it is also a direct consequence of [14, Théorème de l'élément primitif].

Corollary 2.3. Let $R / K$ be a Galois extension. If $[R: K] \leq|K|$ then $R / K$ is simple.

Proof. Let $b=[R: K] \leq|K|=p^{s}$ and $a=b / l(R)$. Then $s \geq 1$. By Remark 1.5 , there holds either

$$
l(R)=b \leq b s / \log _{p} b=b s /\left(\log _{p} b+\log _{p}(1+\varepsilon(a, b))\right)
$$

or

$$
l(R) \leq b / 2 \leq b s /(s+1) \leq b s /\left(\log _{p} b+\log _{p}(1+\varepsilon(a, b))\right) .
$$

Whence $R / K$ is simple in virtue of Theorem 2.2.

Example 2.4. By $[4,7]$, we see that there exists a $G$-Galois extension $R / G F(q)$ satisfying $q=5,|G|=72$, and $l(R)=12$. Put $b=|G|$ and $a=b / l(R)$. Then the equation $(* *)$ in Theorem 1.4 coincides with that in Example 1.6. Using this fact, we know that the right-hand side in the inequality of Theorem 2.2 is more than 26.1 and less than 26.2 . Hence $R / G F(q)$ is simple by Theorem 2.2. On the other hand, there exists a $G$-Galois extension $R / G F(q)$ such that $q=5,|G|=6^{6}$, and $l(R)=6^{5}$. Then, by a direct computation, we see that $l(R)>b s /\left(\log _{p} b+\log _{p}(1+\varepsilon(a, b))\right)$. Hence, in this case, $R / \mathrm{GF}(q)$ is not simple.

\section{ACKNOWLEDGMENT}

The authors would like to express their gratitude to Professor K. Motose for his helpful suggestions.

\section{REFERENCES}

1. S. U. Chase, D. K. Harrison, and Alex Rosenberg, Galois theory and Galois cohomology of commutative rings, Mem. Amer. Math. Soc., no. 52, Amer. Math. Soc., Providence, RI, 1965, pp. 15-33.

2. F. Demeyer and E. Ingraham, Separable algebras over commutative rings, Lecture Notes in Math., vol. 181, Springer-Verlag, Berlin, Heidelberg, and New York, 1971. 
3. G. J. Janusz, Separable algebras over commutative rings, Trans. Amer. Math. Soc. 122 (1966), 461-479.

4. I. Kikumasa, On primitive elements of Galois extensions of commutative semi-local rings. II, Math. J. Okayama Univ. 31 (1989), 57-71.

5. I. Kikumasa and T. Nagahara, On primitive elements of Galois extensions of finite commutative algebras, Math. J. Okayama Univ. 32 (1990), 13-24.

6. _ Primitive elements of cyclic extensions of commutative rings, Math. J. Okayama Univ. 29 (1987), 91-102.

7. I. Kikumasa, T. Nagahara, and K. Kishimoto, On primitive elements of Galois extensions of commutative semi-local rings, Math. J. Okayama Univ. 31 (1989), 31-55.

8. K. Kishimoto, Notes on biquadratic cyclic extensions of a commutative ring, Math. J. Okayama Univ. 28 (1986), 15-20.

9. R. Lidl and Niederreiter, Finite fields, Encyclopedia Math. Appl., vol. 20, Addison-Wesley, Reading, Massachusetts, 1983.

10. T. Nagahara, On separable polynomials over a commutative ring. II, Math. J. Okayama Univ. 15 (1972), 189-197.

11. T. Nagahara and A. Nakajima, On cyclic extensions of commuative rings, Math. J. Okayama Univ. 15 (1971), 81-90.

12. _ On separable polynomials over a commutative ring. IV, Math. J. Okayama Univ. 17 (1974), 49-58.

13. R. S. Pierce, Associative algebras, Graduate Texts in Math., vol. 88, Springer-Verlag, Berlin, Heidelberg, and New York, 1982.

14. J. -D. Thérond, Le théorème de l'élmént primitif pour un anneau semi-local, J. Algebra 105 (1987), 29-39.

15. O. Villamayor and D. Zelinsky, Galois theory for rings with finitely many idempotents, Nagoya Math. J. 27 (1966), 721-731.

16. P. Wolf, Algebraische theorie der Galoisschen algebren, VEB Deutscher Verlag der Wissenschaften, Berlin, 1956.

Department of Mathematics, Okayama University, Okayama 700, Japan 